\title{
PREDICTORS OF POSTOPERATIVE CARDIAC EVENTS AFTER OPEN SURGICAL REPAIR OF ABDOMINAL AORTIC ANEURYSMS
}

\author{
Alonso, A.; Sarrais, C.; Hernández, MM.; Sánchez, JJ.; Martínez, I.; Aguilar, C. \\ Deparment of Anestesiology, Hospital Universitario Clínico San Carlos
}

BACKGOUND: Cardiac complications are the main cause of morbimortality after aortic surgery. Patients subjected to this type of intervention are considered to be high risk of suffering cardiac events during surgery. However, the application of systematic preoperative cardiac screening has been disputed. OBJECTIVES: To identify predictive factors of postoperative cardiac events in our population, and to establish the clinical applicability of preoperative cardiac screening.

MATERIAL AND METHODS: Retrospective review of patients undergoing elective open surgical repair for abdominal aortic aneurysm between $2000-2014$ Main end-point: 30-dav cardiac events : Angina, Heart failure, Cardiogenic shock, Myocardial infarction.

RESULTS: The main data and intraoperative factors of the 542 are shown in the graphics below:

\begin{tabular}{|l|l|}
\hline CARACTERISTICS & \\
\hline Age, years & $68,5+/-7$ \\
Men & $524(96,7 \%)$ \\
\hline Arterial hypertension & $395(72,9 \%)$ \\
\hline Diabetes mellitus & $69(12,7 \%)$ \\
\hline Insulin therapy & $51(9,4 \%)$ \\
\hline Oral Therapy & $18(3,3 \%)$ \\
\hline No smokers & $50(9,2 \%)$ \\
\hline Quitters & $376(69,4 \%)$ \\
\hline Current smokers & $116(21,4 \%)$ \\
\hline Hyperlipidemia & $266(49,1 \%)$ \\
\hline CKD & $79(14,6 \%)$ \\
\hline COPD & $111(20,5 \%)$ \\
\hline Stroke & $37(6,8 \%)$ \\
\hline Pulmonar edema & $103(19 \%)$ \\
\hline
\end{tabular}

\begin{tabular}{|l|l|}
\hline $\begin{array}{l}\text { Previous ischemic } \\
\text { heart disease }\end{array}$ & $135(24,9 \%)$ \\
\hline $\begin{array}{l}\text { Previous } \\
\text { revascularization. }\end{array}$ & $82(15,1 \%)$ \\
\hline $\begin{array}{l}\text { Time since } \\
\text { revascularization }\end{array}$ & \\
$<1$ year & $15(2,76 \%)$ \\
$>1$ year & $67(12,3 \%)$ \\
\hline Atrial fibrillation & $27(5 \%)$ \\
\hline
\end{tabular}

\begin{tabular}{|c|c|}
\hline \multicolumn{2}{|c|}{$\begin{array}{l}\text { Intraoperative } \\
\text { factors }\end{array}$} \\
\hline \multicolumn{2}{|c|}{ Kind of aneurysm } \\
\hline Juxtarenal & $416(76,8 \%)$ \\
\hline Infrarenal & $126(23,2 \%)$ \\
\hline \multicolumn{2}{|c|}{ Kind of clamp } \\
\hline Suprarenal & $136(25,1 \%)$ \\
\hline Infrarenal & $406(74,9 \%)$ \\
\hline
\end{tabular}

97.2\% of patients underwent preoperative cardiac screening: 446(84,6\%): STRESS ECHOCARDIOGRAPHY $32(6,07 \%)$ : ECHOCARDIOGRAPHY I(7,77\%): EXCERCISETREADMILTEST 8(1,52\%):SPECT-MIB
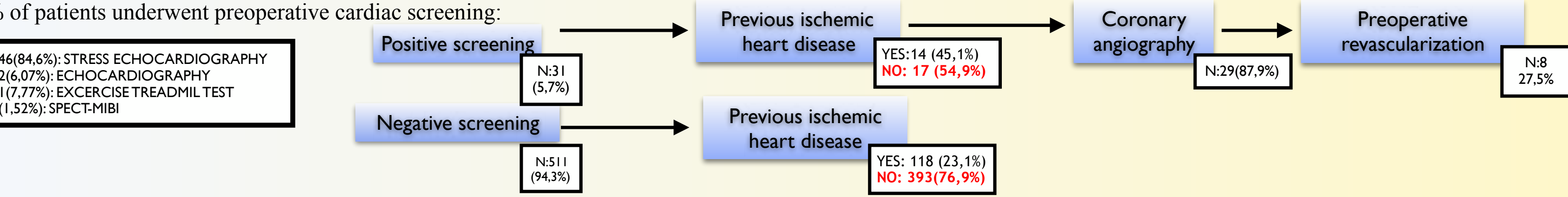

The results of the univariable and multivariable analysis are shown in figure 1 and figure 2 respectively:

\begin{tabular}{|c|c|c|}
\hline Factors & OR (IC95\%) & $\mathbf{P}$ \\
\hline Men & $0,79(0,1-6,16)$ & 0,8 \\
\hline Arterial hypertension & $1,17(0,54-2,54)$ & 0,69 \\
\hline Hyperlipidemia & $1,77(0,89-3,52)$ & 0,09 \\
\hline Current smokers & $2,14(1,04-4,29)$ & 0,035 \\
\hline Diabetes & $2,34(1,07-5,29)$ & 0,04 \\
\hline Age $>65$ years & $3,42(1,19-9,82)$ & 0,016 \\
\hline CKD & $1,4(0,59-3,32)$ & 0,44 \\
\hline COPD & $1,48(0,69-3,16)$ & 0,31 \\
\hline Stroke & $1,22(0,38-1,9)$ & 0,73 \\
\hline Pulmonar edema & $1,4(0,64-3,1)$ & 0,39 \\
\hline $\begin{array}{l}\text { Previous ischemic } \\
\text { heart disease }\end{array}$ & $2,19(1,1-4,35)$ & 0,023 \\
\hline $\begin{array}{l}\text { Ejection fraction } \\
<60 \%\end{array}$ & $2,34(1,01-5,41)$ & 0,05 \\
\hline $\begin{array}{l}\text { Positive cardiologic } \\
\text { screening }\end{array}$ & $2,88(1,04-7,99)$ & 0,05 \\
\hline $\begin{array}{l}\text { Aortic diameter }>\text { or }= \\
70 \mathrm{~mm}\end{array}$ & $2,48(1,24-4,94)$ & 0,008 \\
\hline Juxtarenal aneurysm & $1,43(0,67-2,99)$ & 0,33 \\
\hline Suprarenal clamping & $1,47(0,72-3,02)$ & 0,27 \\
\hline
\end{tabular}

\begin{tabular}{|c|c|c|}
\hline \multicolumn{3}{|c|}{ Independent predictors of postoperative cardiac events } \\
\hline PREDICTORS & OR (Cl) & $p$ \\
\hline AGE $>65 \mathrm{Y}$ & $3,4(1,16-10,02)$ & 0,01 \\
\hline $\begin{array}{c}\text { AORTIC DIAMETER }>\text { OR } \\
=70 M M\end{array}$ & $2,24(1,09-4,58)$ & 0,03 \\
\hline DIABETES & $2,56(1,11-5,92)$ & 0,03 \\
\hline CURRENT SMOKERS & $2,13(1,02-4,45)$ & 0,05 \\
\hline $\begin{array}{l}\text { POSITIVE CARDIAC } \\
\text { SCREENING }\end{array}$ & $2,82(1-8,21)$ & 0,05 \\
\hline
\end{tabular}

Figure 2

Postoperative cardiac events:

Positive screening (n:31): $5(16,1 \%) \quad$ OR 2,88 $(1,04-7,99)$

Negative screening (n:511): $32(6,3 \%)$

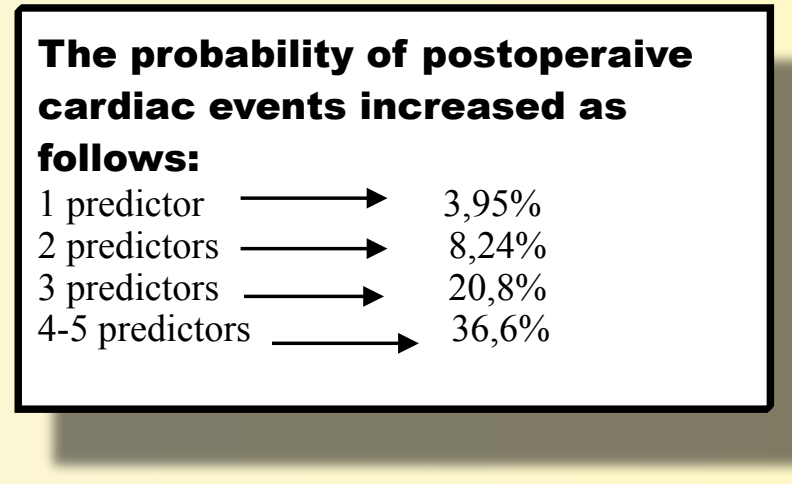

The probability of postoperaive (ancreased as

$(0,72-3,02)$

0,27

CONCLUSION:Postoperative cardiac events after aortic surgery are low in our serie as well as prevalence of coronary artery disease detected by the screening protocol. Although risk subgroups have been identified, systematic cardiac screening is associated with low cardiac morbimortality rates. 